\title{
Indirect Treatment Comparison of Damoctocog Alfa Pegol versus Turoctocog Alfa Pegol as Prophylactic Treatment in Patients with Hemophilia A
}

\author{
Parth Vashi ${ }^{1, *}$ \\ Katharine $\mathrm{Batt}^{2, *}$ \\ Robert Klamroth (iD ${ }^{3}$ \\ Maria Elisa Mancuso ${ }^{4}$ \\ Renata Majewska iD ${ }^{5,6}$ \\ Andreas Tiede (iD) ${ }^{7}$ \\ Lorenzo Giovanni Mantovani ${ }^{8,9}$ \\ 'US Data Generation and Observational \\ Studies, Bayer Corporation, Whippany, \\ NJ, USA; ' 2 Department of Internal \\ Medicine, Section on Hematology/ \\ Medical Oncology, Wake Forest \\ University Baptist Medical Center, \\ Winston-Salem, NC, USA; ${ }^{3}$ Department \\ for Internal Medicine - Vascular Medicine \\ and Coagulation Disorders, Vivantes \\ Klinikum im Friedrichshain, Berlin, \\ Germany; ${ }^{4}$ Center for Thrombosis and \\ Hemorrhagic Diseases, IRCCS Humanitas \\ Research Hospital, Rozzano, Milan, Italy; \\ ${ }^{5}$ HEOR, Creativ-Ceutical, Krakow, \\ Poland; ${ }^{6}$ Department of Epidemiology, \\ Epidemiology and Preventive Medicine, \\ Jagiellonian University Medical College, \\ Krakow, Poland; ${ }^{7}$ Hematology, \\ Hemostasis, Oncology and Stem Cell \\ Transplantation, Hannover Medical \\ School, Hannover, Germany; ${ }^{8}$ Center for \\ Public Health Research, University of \\ Milan - Bicocca, Monza, Italy; ${ }^{9}$ Value- \\ Based Healthcare Unit, IRCCS \\ Multimedica, Sesto San Giovanni, Italy \\ *These authors contributed equally to \\ this work
}

Correspondence: Renata Majewska Creativ-Ceutical, ul. Przemysłowa 12, Kraków, 30-70I, Poland

Tel +33176704790

Email renata.majewska@creativ-ceutical. com
Purpose: To assess the efficacy and FVIII consumption of BAY 94-9027 versus N8-GP in prophylaxis in adolescent and adult patients with severe hemophilia A (HA).

Patients and Methods: A systematic literature review was conducted to identify studies on the efficacy of BAY-94-9027 and N8-GP for prophylaxis in patients with HA aged $\geq 12$ years without a history of inhibitors. Eight studies met systematic literature review inclusion criteria, but only data from PROTECT VIII on BAY 94-9027 and PATHFINDER 2 on N8GP could be used for an indirect comparison. Matching-adjusted indirect comparison (MAIC) and simulated treatment comparison were performed.

Results: No significant differences (unadjusted and adjusted) were observed in the mean annualized bleeding rate (ABR) for any bleed and proportion of patients with zero bleeds when comparing BAY 94-9027 to N8-GP. The adjusted treatment difference [incidence rate ratio (IRR)] in terms of ABR was 1.11 (95\% CI, 0.85-1.44). The odds ratio (OR) of any bleed, measuring the relative effect of BAY 94-9027 versus N8-GP on the proportion of patients with zero bleeds, was 1.03 (95\% CI, 0.60-1.77). FVIII consumption was significantly lower in BAY 94-9027 [mean adjusted difference=-1292.57 IU/kg/year (95\% CI, 2152.44 to -432.70$)$ ]; a 26.7\% reduction in consumption of BAY-94-9027. The results of the sensitivity analyses were similar to the main analysis for mean ABRs, percentages of patients with zero bleeds, and significant reduction in rFVIII consumption. For patients on BAY 949027 every-5-days and every-7-days, no differences versus every-4-days N8-GP were observed for the mean ABR for any bleed [IRR $=0.90(95 \% \mathrm{CI}, 0.68-1.20)]$ and proportion of patients with zero bleeds [OR=1.06 (95\% CI, 0.56-2.02)].

Conclusion: BAY 94-9027 prophylaxis demonstrated $26.7 \%$ lower annual consumption when compared to N8-GP with similar efficacy in terms of ABR and percentage of patients with zero bleeds.

Keywords: BAY 94-9027, N8-GP, prophylaxis, bleed, coagulation factor VIII, adults, factor VIII consumption

\section{Introduction}

The current standard of care in patients with hemophilia (HA) is a regular replacement of or prophylactic treatment with coagulation factor VIII using either recombinant (rFVIII) or plasma-derived (pdFVIII) clotting factor concentrates intended to prevent recurrent bleeds and subsequent joint damage. ${ }^{1,2}$ Patients on prophylaxis have been shown to require fewer hospital admissions and surgical interventions, less frequent unscheduled visits for breakthrough bleeds, and report better quality of life and less time off school/ work. ${ }^{1}$ 
Although hemophilia is a rare disease, it is associated with high aggregate costs and imposes a high financial burden on individuals, healthcare systems, and society in general. Hemophilia is a chronic condition that requires lifelong treatment, with individual costs varying based on disease severity, complications, and treatment regimen. ${ }^{3}$ Medications to treat hemophilia cost more than $\$ 270,000$ annually per patient on average in the US. ${ }^{4}$ If complications occur, the annualized cost can exceed $\$ 1$ million. ${ }^{5}$

The latest advances in product development aim to extend the half-life of FVIII using fusion to protein conjugates (Fc part of IgG1 or albumin), chemical modification (PEGylation), and protein sequence modification. ${ }^{6}$ Extended half-life rFVIII products, such as damoctocog alfa pegol (BAY 94-9027, Jivi, Bayer AG, Berkeley, USA), have decreased the frequency of infusions from about three to one to two per week. ${ }^{7,8}$ BAY 949027 effectively prevented and treated bleeds with individually tailored dose regimens at intervals up to every 7 days. ${ }^{8}$ Little is known about the comparative efficacy of different extended half-life (EHL) rFVIII products. There are no headto-head efficacy trials, and the comparison is even more difficult because of lack of randomized trials with a common comparator arm. A comparison of BAY 94-9027 versus two EHL rFVIII agents (efmoroctocog alfa (rFVIIIFc), Elocta from Sobi, Orphan Biovitrum AB, Stockholm, Sweden and rurioctocog alfa pegol (BAX 855), Adynovate/ Adynovi, Takeda, Lexington, MA, USA) for prophylaxis in patients with severe HA was previously performed using the method of matching-adjusted indirect comparison (MAIC). ${ }^{9}$ The results showed that a mean FVIII consumption was over 20\% lower with BAY 94-9027 than with rFVIIIFc and median consumption tended to be lower with BAY 94-9027 than with BAX 855. Furthermore, mean annualized bleeding rates (ABRs) and percentages of patients with zero bleeds were similar between BAY 94-9027 and comparators. Prophylaxis with BAY-94-9027 and turoctocog alfa pegol (N8-GP, Esperoct, Novo Nordisk, Bagsvaerd, Denmark) has not been compared yet as N8-GP was not yet available (FDA approval received Feb $2019^{10}$ ). Therefore, we conducted this analysis to assess the efficacy and FVIII consumption of those agents in adolescent and adult patients with severe HA based on existing clinical trial data using the same methodology as earlier.

\section{Materials and Methods}

\section{Data Sources and Feasibility Analysis}

A systematic literature review was conducted to identify relevant sources of data. The objective of the systematic literature review was to identify all clinical evidence in patients with HA aged $\geq 12$ years regarding the efficacy of BAY-94-9027 and N8-GP for prophylaxis in patients without a history of inhibitor. Searches in key databases (MEDLINE and MEDLINE In-Process, EMBASE and Cochrane Library) were performed on December 3, 2019 and November 18, 2019. Additionally, a hand search of ClinicalTrials.gov was performed to identify other relevant data. The search was restricted to full-text articles.

After screening titles and abstracts of 76 publications, 7 were included in the full-text review. A total of 5 studies $(6$ records) met the final inclusion criteria and were included in the systematic literature review. Three studies assessed BAY 94-9027: the PROTECT VIII study ${ }^{8}$ was partially randomized, an extension of the PROTECT VIII ${ }^{11}$ study was an open-label study, and Phase I trial ${ }^{12}$ was nonrandomized. Three studies assessed N8-GP (the PATHFINDER 2 study ${ }^{13}$ was partially randomized, but the two follow-ups on some patients who completed the PATHFINDER 2 study ${ }^{14}$ were nonrandomized). As the baseline characteristics of patients continuing in the extension phase of PATHFINDER-2 were not available, extension studies were excluded. It was not possible to adjust for differences in characteristics of patients between extension studies. Additionally, outcomes were reported in the PATHFINDER 2 extension publication by regimen, with patients contributing to several regimens if they switched. Therefore, if we used data by regimen, there would be an additional problem of comparability due to lack of data on periods of time for which patients were observed on different regimens. If we used pooled data from different regimens, then some patients would be double counted. The phase I trial was excluded as well.

In summary, the analysis was based on the main phases of two clinical trials: PROTECT VIII on BAY 94-9027 and PATHFINDER 2 on N8-GP. The PROTECT VIII study is an open-label Phase II/III trial of parallel design with one ondemand and three prophylaxis arms (different schedules and doses) and nonrandomized allocation between on-demand and prophylaxis arms. The PATHFINDER 2 trial on N8-GP is an open-label, nonrandomized Phase III trial of parallel design with one on-demand and one prophylaxis arm. Both studies had similar inclusion criteria (indication, age range, length of previous treatment), but there were differences in characteristics of included populations. The reported outcomes were defined similarly in the PROTECT VIII and PATHFINDER 2 studies: annualized medians and means of any treated bleeds and medians of spontaneous bleeds and rates of patients with 
no bleeds during the study. Moreover, both studies reported a mean annualized consumption in consistent units (IU/kg). The length of the main phase observation period was 36 weeks in the PROTECT VIII trial and approximately 1 year in the PATHFINDER 2 trial. Individual patient data (IPD) for the PROTECT VIII trial were provided by Bayer. For the PATHFINDER 2 trial, only aggregate study-level information was available.

\section{Study Outcomes}

The primary outcomes of interest were ABRs (any bleeds) and annualized consumption of factor VIII (IU/kg/y). Due to different observation periods between compared studies, the proportion of patients with zero bleeds was considered as a secondary outcome only.

\section{Statistical Analyses}

Although both PROTECT VIII and PATHFINDER 2 trials had control arms (on-demand arms), methods for unanchored (without a common comparator) trials were applied, ie, the data from on-demand arms were not considered. Patients were not randomly allocated to on-demand arms; therefore, the outcomes observed in prophylaxis and on-demand arms were not comparable. MAIC and regression methods [simulated treatment comparison (STC)] - both recommended by the National Institute for Health and Care Excellence Decision Support Unit for indirect treatment comparison (ITC) of nonrandomized trials - were applied. ${ }^{15}$ MAIC was chosen as the primary scenario as it uses fewer assumptions regarding the type of relationship between adjustment variables and outcomes. The purpose of a MAIC is to reduce bias caused by differences in the characteristics of the populations (eg, baseline demographic characteristic, disease severity, or prior treatments) of included trials.

In this analysis, MAIC was implemented in the following steps. First, a weight was assigned to each participant of the PROTECT VIII study such that the weighted studylevel baseline characteristics of PROTECT VIII reflected the baseline characteristics of the PATHFINDER 2 study. Second, the parameters measuring the effect of BAY 949027 (as mean of ABR) were estimated using the assigned weights such that the greater a weight assigned to a patient, the higher the impact on the estimated treatment effect. In each of these two steps IPD of PROTECT VIII was used. Finally, a relative effect of BAY 94-9027 compared to N8-GP was estimated using the weighted effect of BAY 94-9027, as estimated in the previous steps of the MAIC, and the effect of N8-GP as reported in publications of the PATHFINDER 2 study. This process is illustrated in Appendix Figure 1.

Outcomes of interest were compared between trials in planned prophylaxis treatment groups [intention-to treat (ITT)].

Primary analyses focused on a comparison of ABR and consumption using pooled prophylaxis arms in the PROTECT VIII study. Prophylaxis arms [twice weekly $(n=24)$, every 5 days $(n=43)$, and every 7 days $(n=43)]$ were assumed comparable and pooled into one prophylaxis group, as there were no significant differences in outcomes of interest between groups. Prophylaxis primary efficacy outcome (ABR) as well as a mean annualized total FVIII consumption (IU/kg/year) were based on data from weeks 11 to 36 (end of run-in/randomization to end of the main phase). The same rule was applied to the percentage of patients with zero bleeds, a secondary efficacy outcome. Patients in the prophylaxis arm were initially treated with $25 \mathrm{IU} / \mathrm{kg}$ BAY 94-9027 twice weekly during a 10-week run-in period, which was used to identify patients who experienced more frequent bleed and therefore were not expected to benefit from less frequent infusions and were therefore not randomized. ${ }^{8}$

To assess the mean ABR for any bleed, a Poisson regression model on the number of bleeds per patient, allowing for overdispersion using imputed ABR for missing data was used in the PATHFINDER 2 study. ${ }^{13}$ The same methodology was applied to the patient-level ABR data from the PROTECT VIII study. The following covariates available in both trials were considered for population adjustment and weight calculations: age (years); race (white/Asian/other), weight $(\mathrm{kg})$, prior prophylaxis treatment (yes/no), prior number of bleeds $>2$ in prior prophylaxis treatment, and prior number of bleeds $>19$ in prior on-demand treatment.

Relative treatment effects, comparing BAY 94-9027 vs N8-GP for assessed outcomes, and associated 95\% confidence intervals (CI) were estimated for both weighted (adjusted comparison) and unweighted (naïve comparison) estimates from the IPD analysis of PROTECT VIII compared to previously published estimates from PATHFINDER 2:

- Incidence rate ratio (IRR) with 95\% CI was reported for ABR for any bleeds,

- Odds ratio (OR) with $95 \%$ CI was reported for the percentage of patients without bleeding events, 
- Mean differences with 95\% CI were reported for annualized consumption.

All calculations were done using R 4.0.2.

\section{Sensitivity Analysis}

Four sensitivity analyses were conducted for primary and secondary outcomes to test the robustness of the results for the comparison between BAY-94-9027 and N8-GP used as prophylactic treatment in patients with severe HA. Sensitivity analysis 1 was an ITC analysis using STC with all pooled prophylaxis arms of the PROTECT VIII trial. Sensitivity analysis 2 was a MAIC analysis using ITC between the prophylaxis arm of the PATHFINDER 2 study and pooled every-5-days and every-7-days prophylaxis arms of the PROTECT VIII study. Sensitivity analysis 3 was a MAIC analysis using ITC between the prophylactic arm of the PATHFINDER 2 study and pooled biweekly and every5-days prophylaxis arms of the PROTECT VIII study. In the last analysis, we compared all outcomes of interest including the run-in period; therefore, outcomes were summarized from week 0 to 36 for all pooled prophylaxis arms of the PROTECT VIII trial.

\section{Results}

\section{Baseline Characteristics and Effective Sample Size}

Baseline characteristics before and after matching of all variables simultaneously (main scenario) are presented in Table 1. Before matching, the mean age of patients from the PROTECT VIII study was higher than that of the PATHFINDER 2 study $(\mathrm{P}<0.01)$. There were also higher percentages of patients of Asian race with number of bleeds $>19$ in the prior on-demand treatment group in the PROTECT VIII study compared to the PATHFINDER 2 study ( $\mathrm{P}>0.05$ and $\mathrm{P}<0.05$, respectively).

The post-matching characteristics in the PROTECT VIII study were comparable to those in the PATHFINDER 2 study. The effective sample size of the PROTECT VIII study was 61.94 (60.73\% of the original sample of 102 patients).

\section{ITC of BAY 94-9027 vs N8-GP}

Adjusted estimates for mean ABR for any bleed were close to the crude mean ABR number reported in the PROTECT VIII trial (Figure 1). No statistically significant differences were observed in the mean $\mathrm{ABR}$, primary efficacy outcomes when comparing BAY 94-9027 to N8-GP, nor in unadjusted or adjusted analyses. The adjusted treatment difference between BAY 94-9027 to N8-GP considering ABRs, ie the IRR of bleed, was 1.11 (95\% CI, 0.85-1.44). Statistically significant differences were observed in factor VIII consumption, noted lower in BAY 94-9027 compared to N8GP with the mean adjusted difference of $-1292.57 \mathrm{IU} / \mathrm{kg} / \mathrm{y}$ (95\% CI, -2152.44 to -432.70 ). This difference reflects a $26.7 \%$ reduction in the units consumption of BAY-949027 compared to N8-GP. A lack of significant difference was also observed for the proportion of patients without bleeds when comparing BAY 94-9027 to N8-GP. The OR of any bleed, measuring the relative effect of BAY 94-9027 versus N8-GP on the proportion of patients with zero bleeds was 1.03 (95\% CI, 0.60-1.77). Moreover, the MAICadjusted median ABR for spontaneous bleeds were the same between compared treatments and equaled zero.

\section{Sensitivity Analysis}

The results of the sensitivity analyses were similar to the main analysis with no statistical difference in mean ABRs and a statistically significant reduction in rFVIII consumption. The adjusted treatment effect in terms of ABR, ie the IRR of bleed, was 0.88 (95\% CI, 0.66-1.16) using the STC method instead of MAIC, 0.90 (95\% CI, 0.68-1.20) when pooling only patients treated with every 5 days and every 7 days regimens, 1.09 (95\% CI: 0.02-66.36) when pooling those treated with biweekly and every 5 days regimens for BAY 94-9027 and 1.11 (95\% CI 0.86-1.45) when pooling all prophylaxis arms and including run-in period (Table 2). The STC-adjusted mean annualized consumption was significantly lower in the PROTECT VIII study compared to the PATHFINDER 2 study with an adjusted difference of $1336.31 \mathrm{IU} / \mathrm{kg} /$ year (95\% CI, -2064.74 to -607.88 ). A similar difference in consumption was observed when pooling patients treated with every 5 days and every 7 days regimens for BAY 94-9027; (adjusted difference of -1453.26 (95\% CI, -2291.62 to -614.91$) \mathrm{IU} / \mathrm{kg} /$ year and when pooled biweekly and every 5 days prophylaxis PROTECT VIII arms were used (adjusted difference of $-1137.62 \mathrm{IU} / \mathrm{kg} /$ year $(95 \%$ CI, -1855.45 to -419.79 ), see Table 2 .

The results of the sensitivity analyses were also similar when the secondary outcome was considered. No statistically significant difference in the proportion of patients with zero bleeds between BAY 94-9027, and N8-GP was observed for none of the sensitivity analysis scenario (Table 2). 
Table I Quality of Baseline Characteristics Matching (Main Scenario)

\begin{tabular}{|c|c|c|c|c|c|}
\hline \multirow[t]{2}{*}{ Variables } & \multicolumn{3}{|c|}{ Prematching Characteristics } & \multicolumn{2}{|c|}{ Postmatching Characteristics } \\
\hline & PATHFINDER 2 & PROTECT VIII (IPD) & $P$ value & PROTECT VIII & $P$ value \\
\hline N & 175 & $102 *$ & & $61.94(60.73 \%)$ & \\
\hline Age, mean [years] (SD) & $30.60(12.50)$ & 34.54 (I2.95) & 0.0021 & $30.60(12.49)$ & 1.000 \\
\hline Weight, mean [kg] (SD) & $75.00(14.40)$ & $76.74(17.15)$ & 0.3652 & $75.00(14.40)$ & 1.000 \\
\hline Asian race $(\%)$ & 18 & 26 & 0.1152 & 18 & 1.000 \\
\hline White race (\%) & 77 & 71 & 0.2682 & 77 & 1.000 \\
\hline Prior PPX (\%) & 85 & 80 & 0.2844 & 85 & 1.000 \\
\hline Prior no. of bleeds $>2$ in prior PPX (\%) & 50 & 57 & 0.3090 & 50 & 1.000 \\
\hline Prior no. of bleedings $>19$ in prior OD (\%) & 50 & 85 & 0.0145 & 50 & 1.000 \\
\hline
\end{tabular}

Notes: *Eight patients had missing values for at least one variable used for matching.

Abbreviations: IPD, individual patient data; OD, on-demand treatment; PPX, prophylaxis.

\section{Discussion}

HA is a rare disease, which may explain why no head-tohead efficacy studies comparing active treatments were identified in the systematic literature review. For ethical reasons, existing studies are nonrandomized, and moreover, patients with a prophylaxis history should not be allocated to on-demand arms. Naive, unanchored, comparisons of results observed in different treatments in different studies are of low credibility due to many confounding factors (eg, population, outcomes, study design). Methods such as MAIC and STC used for this analysis may reduce bias related to known differences between study populations because the population of one study is adjusted to reflect the population of the other one.

According to the previous MAICs, ${ }^{9}$ ABR (considered the most clinically meaningful efficacy endpoint ${ }^{16}$ ) and percentages of patients with zero bleeds were similar were similar comparing BAY 94-9027 with rFVIIIFc, BAX 855, and rAHF-PFM. (Appendix Table 1). A recent MAIC with $\mathrm{rFVIIIFc}^{17}$ suggested no difference in the proportion of patients with zero bleeds between individualized rFVIIIFc and BAY 94-9027 pooled prophylaxis arms, but a significantly lower mean $A B R$ in the rFVIIIFc individualized prophylaxis group versus the BAY 94-9027 pooled prophylaxis population (mean difference $[\mathrm{MD}]-1.9 ; 95 \%$ confidence interval $[\mathrm{CI}]-3.5$ to -0.4). In the publication by Batt et $\mathrm{al},{ }^{9}$ the mean ABR after weighting for difference in baseline characteristics was similar between BAY 94-9027 and individualized rFVIIIFc: 4.25 vs $2.91(P>0.05)$. The difference in the results might be explained by different set of variables used in weighting of individualized data, population selection, as well as different summary measures compared.
The publication of Hakimi et $\mathrm{al}^{17}$ has a relatively high effective sample size, which helps reaching statistical significance, but this was associated with the use of fewer adjustment factors. There was no adjustment on prior use of prophylaxis. Additionally, the population was selected to be specific to only one of the treatment arms studied for rFVIIIFc compared to a pooled analysis of all treatment arms from the PROTECT VIII trial. Furthermore, the recent publication ${ }^{17}$ was based on a comparison of arithmetic means of $\mathrm{ABR}$, while that of Batt et $\mathrm{al}^{9}$ compared mean ABRs based on a negative binomial model. ABR was highly skewed in the PROTECT VIII trial, therefore means based on a negative binomial model would be a better summary here than arithmetic means. The study by Hakimi et al also did not consider the difference in the quantity of medication consumed between rFVIIIFc and BAY 94-9027.

The present analysis demonstrates similar findings for BAY 94-9027 and N8-GP in both ABR (IRR of 1.11 (95\% CI, 0.85-1.44; $p$-value 0.4490)) and number of patients with zero bleeds (1.03 (95\% CI, 0.60-1.77; p-value 0.9039)). As ABR appears to be similar between products, other criteria need to be considered when choosing between products. The quantity of medications used for treating HA and preventing complications correlate with cost and are a key factor. This study showed that the use of medication is over $20 \%$ lower with BAY 94-9027 than with N8-GP, as previously shown vs rFVIIIFc, the reduction of the mean annualized consumption of FVIII was here 26\% (3552.43 IU/ $\mathrm{kg} /$ year vs $4845.00 \mathrm{IU} / \mathrm{kg} / \mathrm{year}$, respectively). This might be considered as an important advantage of BAY 94-9027.

Our main finding of reduced factor consumption while maintaining similar bleed rates has relevance to a clinical 

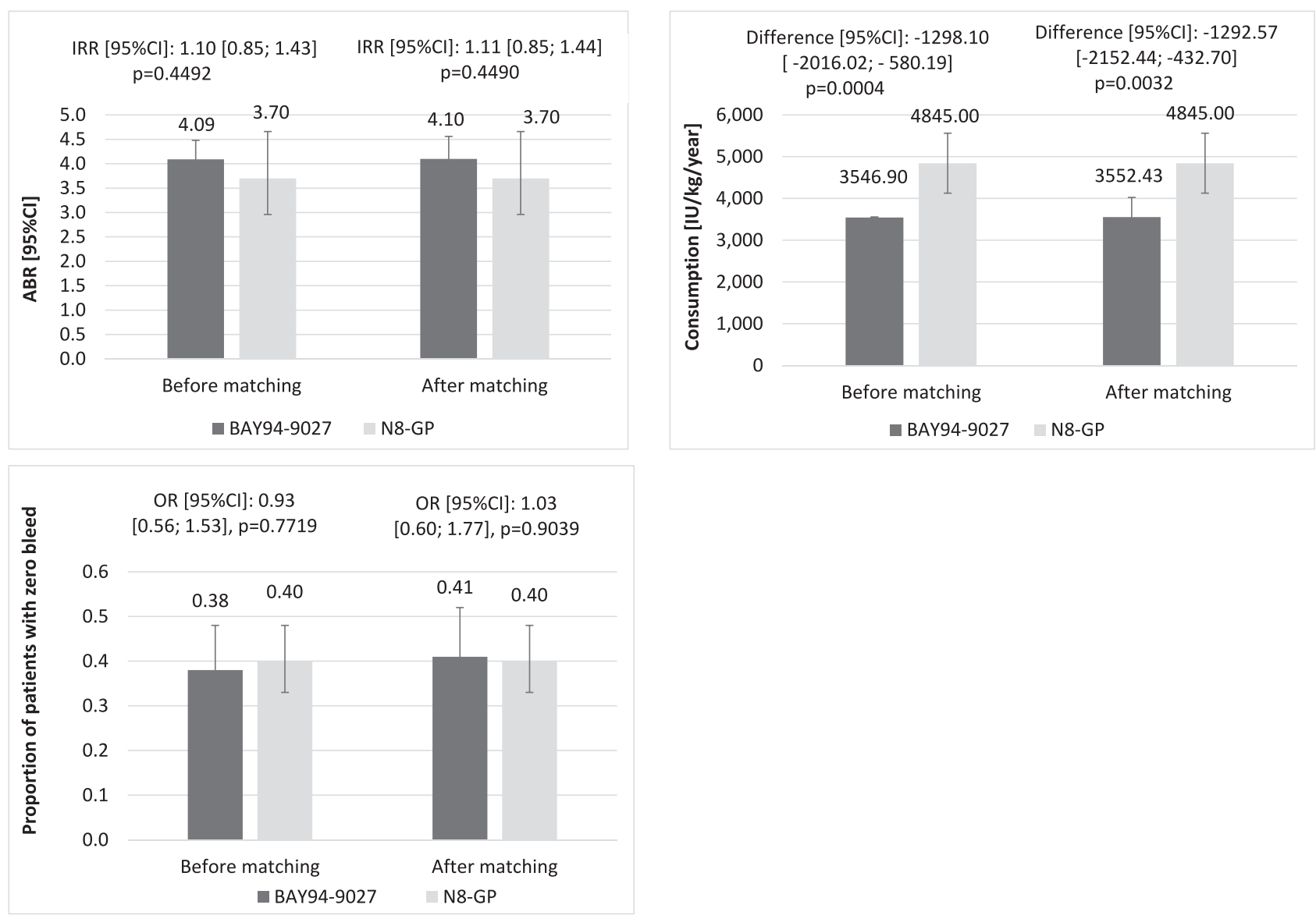

Figure I Comparison of ABR, consumption and proportion of patients with zero bleed between BAY94-0927 and N8-GP. Abbreviations: $\mathrm{ABR}$, annualized bleeding rate; $\mathrm{Cl}$, confidence interval; OR, odds ratio; $\mathrm{P}, \mathrm{P}$-value; IRR, incidence rate ratio.

audience, given the financial burden HA care has on health care budgets. It is therefore important to consider the underlying reasons for our findings and the implications for translation into clinical practice benefits under realworld conditions. First, differences in pharmacokinetics may account in part for the effects observed in our study. Head-to-head pharmacokinetic studies comparing BAY 94-9027 and N8-GP are not available. Compared to the PEGylated rFVIII concentrate, BAX 855, BAY 94-9027 showed significantly increased exposure (area under the curve (AUC) ratio 1.22 [95\% confidence interval 1.111.33], $P=0.0004$ ) due to slower clearance (ratio 0.82 [0.75-0.90], $P=0.0004) .{ }^{18}$ Likewise, compared to rFVIIIFc, BAY 94-9027 showed higher exposure (AUC ratio 1.26 [1.14-1.38], $P=0.0001)$ and slower clearance (ratio 0.80 [0.72-0.87], $P=0.0001$ ). ${ }^{19}$ The differences in FVIII clearance is thought to be linked to the structure of the low-density lipoprotein receptor and size of PEGylation extending the FVIII half-life. Also, the type of conjugation, eg, site-specific conjugation as used in
BAY 94-9027, may contribute to extending the pharmacokinetics and/or pharmacodynamics or change its dynamics. ${ }^{18,20}$ Thus, PEGylation is one of the factors likely explaining the similar $>20 \%$ reduction in factor use when comparing the BAY 90-9027 clinical trials to it's the other factor concentrates. Second, differences in study design may in part explain the reduced factor consumption with BAY 94-9027 as compared with N8-GP as seen in our study. N8-GP was used at the same dosing regimen (50 IU $/ \mathrm{kg}$ every 4 days) for all patients in the PATHFINDER 2 study. In contrast, the PROTECT VIII study had a run-in phase of 10 weeks (BAY 94-9027 dosed $25 \mathrm{IU} / \mathrm{kg}$ twice-weekly) that stratified patients with low bleeding risk ( 0 or 1 breakthrough bleeds, later-on randomized to prophylaxis every 5 or every 7 days) vs higher bleeding risk ( $\geq 2$ breakthrough bleeds, later-on prophylaxis twice-weekly). This stratification resulted in spending more factor concentrate on prophylaxis in patients with higher bleeding risk as compared to patients with lower bleeding risk and finally resulted in similar ABR across all 
Table 2 Indirect Treatment Comparison Between BAY-94-9027 and N8-GP in Prophylaxis Hemophilia Treatment (Sensitivity Analyses)

\begin{tabular}{|c|c|c|c|c|c|}
\hline \multirow[t]{2}{*}{ Variables } & \multirow[t]{2}{*}{ PATHFINDER 2} & \multicolumn{2}{|l|}{ PROTECT VIII } & \multicolumn{2}{|c|}{ Comparison of PROTECT-VIII vs PATHFINDER 2} \\
\hline & & Crude Estimates & $\begin{array}{l}\text { Adjusted } \\
\text { Estimates }\end{array}$ & Crude Estimates & Adjusted Estimates \\
\hline ABR for any bleeds* & Mean $(95 \% \mathrm{Cl})$ & Mean $(95 \% \mathrm{Cl})$ & Mean $(95 \% \mathrm{Cl})$ & IRR (95\% CI); p & IRR (95\% CI); p \\
\hline STC - pooled all PPX arms & $3.7(2.96-4.66)$ & $4.09(3.72-4.48)$ & $3.24(2.8 \mathrm{I}-3.73)$ & 1.10 (0.85-I.43); 0.4492 & 0.88 (0.66-I.16); 0.3490 \\
\hline $\begin{array}{l}\text { MAIC - pooled Q5D and Q7D PPX } \\
\text { arms }\end{array}$ & $3.7(2.96-4.66)$ & $3.81(3.40-4.26)$ & $3.34(2.86-3.90)$ & $1.03(0.79-1.34) ; 0.8351$ & $0.9(0.68-1.20) ; 0.4818$ \\
\hline $\begin{array}{l}\text { MAIC - pooled BIW and Q5D PPX } \\
\text { arms }\end{array}$ & $3.70(2.96-4.66)$ & $4.01(3.54-4.53)$ & $4.02(0.07-243.84)$ & $1.08(0.83-1.42) ; 0.5617$ & $1.09(0.02-66.36) ; 0.9686$ \\
\hline $\begin{array}{l}\text { MAIC - pooled all PPX arms (including } \\
\text { run-in period) }\end{array}$ & $3.70(2.96-4.66)$ & $4.10(3.75-4.50)$ & $4.12(3.70-4.58)$ & I.II (0.86-I.43); 0.4269 & I.II (0.86-I.45); 0.4226 \\
\hline Consumption (IU/kg/year) & Mean $(95 \% \mathrm{Cl})$ & Mean $(95 \% \mathrm{Cl})$ & Mean $(95 \% \mathrm{Cl})$ & Difference (95\% Cl); p & Difference $(95 \% \mathrm{Cl}) ; \mathrm{p}$ \\
\hline STC - pooled all PPX arms & $\begin{array}{l}4845.00(4127.17- \\
5562.83)\end{array}$ & $\begin{array}{l}3546.90(3536.15- \\
3557.64)\end{array}$ & $\begin{array}{l}3508.69(3247.15- \\
3445.14)\end{array}$ & $\begin{array}{l}-1298.1(2016.02 \text { to }-580.19) \\
0.0004\end{array}$ & $\begin{array}{l}-1336.3(-2064.74 \text { to } \\
-607.88) ; 0.0001\end{array}$ \\
\hline $\begin{array}{l}\text { MAIC - pooled Q5D and Q7D PPX } \\
\text { arms }\end{array}$ & $\begin{array}{l}4845(4127.17- \\
5562.83)\end{array}$ & $\begin{array}{l}3436.50(3343.39- \\
3529.61)\end{array}$ & $\begin{array}{l}3391.74(2958.67- \\
3824.81)\end{array}$ & $\begin{array}{l}-1408.5(-2132.34 \text { to } \\
-684.65) ; 0.0001\end{array}$ & $\begin{array}{l}-1453.3(-2291.62 \text { to } \\
-6 \mid 4.91) ; 0.0007\end{array}$ \\
\hline $\begin{array}{l}\text { MAIC - pooled BIW and Q5D PPX } \\
\text { arms }\end{array}$ & $\begin{array}{l}4845(4127.17- \\
5562.83)\end{array}$ & $\begin{array}{l}3681.2(3531.25- \\
3831.16)\end{array}$ & $\begin{array}{l}3707.38(3706.53- \\
3708.23)\end{array}$ & $\begin{array}{l}-1163.8(-1897.12 \text { to } \\
-430.47) ; 0.0019\end{array}$ & $\begin{array}{l}-1137.6(-1855.45 \text { to } \\
-419.79) ; 0.0019\end{array}$ \\
\hline $\begin{array}{l}\text { MAIC - pooled all PPX arms (including } \\
\text { run-in period) }\end{array}$ & $\begin{array}{l}4845(4127.17- \\
5562.83)\end{array}$ & $\begin{array}{l}3353.06(3343.47- \\
3362.65)\end{array}$ & $\begin{array}{l}3341.52(2893.12- \\
3789.93)\end{array}$ & $\begin{array}{l}-1491.94(-2209.84 \text { to } \\
-774.04) ;<0.0001\end{array}$ & $\begin{array}{l}-1503.48(-2349.85 \text { to } \\
-657.10) ; 0.0005\end{array}$ \\
\hline $\begin{array}{l}\text { Proportion of patients with zero } \\
\text { bleed (\%) }\end{array}$ & $\begin{array}{l}\text { Proportion } \\
(95 \% \mathrm{CI})\end{array}$ & $\begin{array}{l}\text { Proportion } \\
(95 \% \mathrm{Cl})\end{array}$ & $\begin{array}{l}\text { Proportion } \\
(95 \% \mathrm{Cl})\end{array}$ & OR (95\% Cl); p & OR (95\% Cl); p \\
\hline STC - pooled all PPX arms & $40(33-48)$ & $38(29-48)$ & $42(32-51)$ & 0.93 (0.56-1.53); 0.7719 & 1.08 (0.66-I.77); 0.7655 \\
\hline $\begin{array}{l}\text { MAIC - pooled Q5D and Q7D PPX } \\
\text { arms }\end{array}$ & $40(33-48)$ & $41(30-52)$ & $41(28-55)$ & $\mathrm{I} .04$ (0.6I-I.80); 0.8779 & 1.06 (0.56-2.02); 0.8587 \\
\hline $\begin{array}{l}\text { MAIC - pooled BIW and Q5D PPX } \\
\text { arms }\end{array}$ & $40(33-48)$ & $38(26-49)$ & $41(0-1)$ & 0.90 (0.50-1.62); 0.7261 & $1.05\left(0.00-19 \times 10^{6}\right) ; 0.9951$ \\
\hline $\begin{array}{l}\text { MAIC - pooled all PPX arms (including } \\
\text { run-in period) }\end{array}$ & $40(33-48)$ & $33(24-42)$ & $35(25-46)$ & 0.73 (0.44-I.2I); 0.2229 & 0.83 (0.48-I.42); 0.4907 \\
\hline
\end{tabular}

Notes: *Based on Poisson model. The effective sample size when pooling Q5D and Q7D PPX arms was 40.31 (5I.68\% of the original sample) and 33.14 (5I.78\% of the original sample) when pooling BIW and Q5D PPX arms of PROTECT VIII study.

Abbreviations: ABR, annualized bleeding rate; BIW, twice weekly, IRR, incidence rate ratio; MAIC, matching-adjusted indirect comparison; OD, odds ratio; PPX, prophylaxis; Q5D, every 5 days; Q7D, every 7 days; STC, simulated treatment comparison.

dosing tiers. Therefore, not only product characteristics but guidance for dosing and individualized regimens based on response to treatment have an impact on the balance between resource utilization and clinical outcomes.

In the absence of randomized studies with an identical comparator, the ITC methodological guidelines allows for the use of the MAIC as a reasonable method for comparing nonrandomized, unanchored trials with adjustments made for patient characteristics at baseline, thereby minimizing the bias of comparing effects from trials with different baseline. ${ }^{15}$ By comparing studies with similar outcome definitions (excluding those from the observation period), we minimized study design. Finally, by performing sensitivity analyses around different pooled treatment regimens and other ITC approach (STC), we confirmed the results as they were in line with those obtained in the main analysis.

The use of MAIC or STC instead of naive (nonadjusted) comparison carries some limitations as it reduces bias only if all key treatment modifiers and prognostic factors are included for an adjustment. In our case, 
a clinically important factor of the prior number of target joints bleeds ${ }^{21,22}$ was not considered as this parameter was not reported in the PATHFINDER 2 study. It is also possible that there are other unknown effect modifiers that were not considered in the analysis. It is notable that MAIC adjusts for differences in patient characteristics only, but not for any difference in the study design or outcome definitions. Furthermore, the main phase of the PATHFINDER 2 study was twice as long as in the PROTECT VIII study, which could have additional implications. First, it could affect the comparison of proportions of patients with zero bleeds and result in a bias in favor of BAY 94-9027, because the longer patients are followed, the more likely they are to experience at least one bleed. This efficacy outcome was considered as secondary endpoint of interest. And second, it may also affect the comparison of $\mathrm{ABR}$ as the frequency of bleeding tends to decrease over time in patients treated on prophylaxis. ${ }^{23}$ The same trend was observed in PROTECT-VIII extension study. Mean ABR (based on negative binomial model) observed at the end of main phase of PROTECT VIII was $4.07,{ }^{9}$ while the one observed in extension study ( 5 years of follow-up) was 3.49. ${ }^{11}$ Therefore, the ABR comparison may be biased against BAY 94-9027. Finally, considered study periods were short, and the study with longer data would provide more robust evidence to changes in ABR.

\section{Conclusions}

Prophylactic treatment with BAY 94-9027 shows similar efficacy in terms of $\mathrm{ABR}$ and percentage of patients with zero bleeds and is consistent with a $26.7 \%$ lower annual factor consumption, suggesting potential cost savings with its use.

\section{Abbreviations}

ABR, annualized bleeding rate; CI, confidence interval; EHL, extended half-life; Fc, fragment crystallizable; FVIII, factor VIII; HA, hemophilia A; Ig, immunoglobulin; IRR, incidence rate ratio; ITC, indirect treatment comparison; IU, International units; OR, odds ratio; SD, standard deviation; STC, simulated treatment comparison.

\section{Ethics Approval and Informed Consent}

Both the PATHFINDER 2 and PROTECT VIII studies were conducted in accordance with the Declaration of Helsinki and local regulations. The protocols were approved by the authorities and the ethics committees of the respective institutions, and signed informed consent was obtained from all patients. Informed consent for this analysis was not required given the deidentified nature of the PROTECT VIII individualized patient-level data, and the use of aggregated previously published data from the PATHFINDER 2 study.

\section{Acknowledgments}

The authors thank Małgorzata Biernikiewicz of CreativCeutical for providing medical writing support in accordance with Good Publication Practice (GPP3) guidelines. LGM receives support from the Italian Ministry of Health - Ricerca Corrente IRCCS Muletimedica.

\section{Author Contributions}

All authors made a significant contribution to the work reported, whether that is in the conception, study design, execution, acquisition of data, analysis and interpretation, or in all these areas; took part in drafting, revising or critically reviewing the article; gave final approval of the version to be published; have agreed on the journal to which the article has been submitted; and agree to be accountable for all aspects of the work.

\section{Funding}

This study was funded by Bayer.

\section{Disclosure}

AT received grants or research support or honoraria for lectures or consultancy in the field of hemophilia from Bayer, Biotest, Chugai, CSL Behring, Novo Nordisk, Octapharma, Pfizer, Roche, SOBI, Takeda. KB has no competing interests; she has consulted with Bayer, HEMA, Takeda, CHEORS, Precisionheor, Kezar and Forma Therapeutics. LGM received personal fees from Bayer in the field of hemophilia and from Roche, Pfizer, Biogen, Takeda outside the field of hemophilia; research grants from Bayer, Roche and Takeda in the field of hemophilia and from Roche, Biogen and Bayer outside the field of hemophilia. PV is an employee of Bayer. RK received honoraria for Advisory boards and presentations from Bayer, Biotest, Biomarin, CSL Behring, Grifols, NovoNordisk, Octapharma, Pfizer, Sanofi, SOBI, Takeda, Uniqure. RM is an employee of Creative-Ceutical, which received funds to conduct the study. MEM has acted as paid consultant/advisor/speaker for Bayer Healthcare, Biomarin, Catalyst 
Bioscience, CSL Behring, Grifols, Kedrion, LFB, Novo Nordisk, Octapharma, Pfizer, Roche, Sobi, Spark Therapeutics, Takeda and UniQure. The authors report no other conflicts of interest in this work.

\section{References}

1. Makris M. Prophylaxis in haemophilia should be life-long. Blood Transfus. 2012;10(2):165-168.

2. Srivastava A, Brewer AK, Mauser-Bunschoten EP, et al. Guidelines for the management of hemophilia. Haemophilia. 2013;19(1):e1-e47.

3. Chen SL. Economic costs of hemophilia and the impact of prophylactic treatment on patient management. Am J Manag Care. 2016;22 (5 Suppl):s126-s133.

4. Report ES. Express scripts 2015 drug trend report; 2016. Available from: https://californiahealthline.org/wp-content/uploads/sites/3/ 2018/02/express-scripts-2015-commercial-dtr.pdf. Accessed October $10,2020$.

5. Medicaid Health Plans of America Center for Best Practices. Clinical brief. Addressing the Needs of members with hemophilia in Medicaid managed care: issues and implications for health plans; 2013. Available from: https://www.hca.wa.gov/assets/program/bdc MHPA_hemophilia_issue_brief_082113.pdf. Accessed October 10, 2020.

6. Graf L. Extended half-life factor VIII and factor IX preparations. Transfus Med Hemotherapy. 2018;45(2):86-91. doi:10.1159/ 000488060

7. Konkle BA, Stasyshyn O, Chowdary P, et al. Pegylated, full-length, recombinant factor VIII for prophylactic and on-demand treatment of severe hemophilia A. Blood. 2015;126(9):1078-1085. doi:10.1182/ blood-2015-03-630897

8. Reding MT, Ng HJ, Poulsen LH, et al. Safety and efficacy of BAY 94-9027, a prolonged-half-life factor VIII. J Thromb Haemost. 2017;15(3):411-419. doi:10.1111/jth.13597

9. Batt K, Gao W, Ayyagari R, et al. Matching-adjusted indirect comparisons of annualized bleeding rate and utilization of BAY 94-9027 versus three recombinant factor VIII agents for prophylaxis in patients with severe hemophilia A. J Blood Med. 2019;10:147-159. doi:10.2147/JBM.S206806

10. U.S. Food and Drug Administration. ESPEROCT (STN: BL 125671); 2019. Available from: https://www.fda.gov/vaccines-bloodbiologics/esperoct. Accessed June 30, 2021.

11. Lalezari S, Reding MT, Pabinger I, et al, BAY 94-9027 prophylaxis is efficacious and well tolerated for up to $>5$ years with extended dosing intervals: PROTECT VIII extension interim results. Haemophilia. 2019;25(6):1011-1019. doi:10.1111/hae.13853

12. Coyle TE, Reding MT, Lin JC, Michaels LA, Shah A, Powell J. Phase I study of BAY 94-9027, a PEGylated B-domain-deleted recombinant factor VIII with an extended half-life, in subjects with hemophilia A. J Thromb Haemost. 2014;12(4):488-496. doi:10.1111/ jth.12506
13. Giangrande P, Andreeva, T., Chowdary, P., Ehrenforth, S., Hanabusa, H., Leebeek, FW, Lentz, SR, Nemes, L., Poulsen, LH, Santagostino, E., You, CW, Clausen, WH, Jönsson, PG, Oldenburg, J. Clinical evaluation of glycoPEGylated recombinant FVIII: efficacy and safety in severe haemophilia A. Thromb Haemost. 2017;117 (2):252-261. doi:10.1160/TH16-06-0444

14. Curry N, Albayrak, C., Escobar, M., Holme, PA, Kearney, S., Klamroth, R., Misgav, M., Négrier, C., Wheeler, A., Santagostino, E., Shima, M., Landorph, A., Tønder, SM, Lentz SR. Once-weekly prophylaxis with glycoPEGylated recombinant factor VIII (N8-GP) in severe haemophilia A: safety and efficacy results from pathfinder 2 (randomized phase III trial). Haemophilia. 2019;25:373-381. doi:10.1111/hae.13712

15. Phillippo D, Ades AE, Dias S, Palmer S, Abrams KR, Welton NJ. Nice DSU Technical Support Document 18: Methods for PopulationAdjusted Indirect Comparisons in Submissions to Nice. NICE DSU; 2016.

16. Keipert C, Müller-Olling M, Gauly F, Arras-Reiter C, Hilger A. Annual bleeding rates: pitfalls of clinical trial outcomes in hemophilia patients. Clin Transl Sci. 2020. doi:10.1111/cts.12794

17. Hakimi Z, Santagostino E, Postma MJ, Nazir J. Recombinant FVIIIFc versus BAY 94-9027 for treatment of patients with haemophilia A: comparative efficacy using a matching adjusted indirect comparison. Adv Ther. 2021;38(2):1263-1274. doi:10.1007/s12325020-01599-1

18. Solms A, Shah A, Berntorp E, et al. Direct comparison of two extended half-life PEGylated recombinant FVIII products: a randomized, crossover pharmacokinetic study in patients with severe hemophilia A. Ann Hematol. 2020;99(11):2689-2698. doi:10.1007/s00277-020-04280-3

19. Shah A, Solms A, Wiegmann S, et al. Direct comparison of two extended-half-life recombinant FVIII products: a randomized, crossover pharmacokinetic study in patients with severe hemophilia A. Ann Hematol. 2019;98(9):2035-2044. doi:10.1007/s00277-01903747-2

20. Mei B, Pan C, Jiang H, et al. Rational design of a fully active, long-acting PEGylated factor VIII for hemophilia A treatment. Blood. 2010;116(2):270-279. doi:10.1182/blood-2009-11-254755

21. Thorat T, Neumann PJ, Chambers JD. Hemophilia burden of disease: a systematic review of the cost-utility literature for hemophilia. J Manag Care Spec Pharm. 2018;24(7):632-642. doi:10.18553/ jmcp.2018.24.7.632

22. Arima K, Gupta S, Gadkari A, et al. Burden of atopic dermatitis in Japanese adults: analysis of data from the 2013 national health and wellness survey. J Dermatol. 2018;45(4):390-396. doi:10.1111/13468138.14218

23. Noone D, O'Mahony B, Prihodova L. A survey of the outcome of prophylaxis, on-demand or combined treatment in 20-35 year old men with severe haemophilia in four European countries. Haemophilia. 2011;17(5):e842-e843.
The Journal of Blood Medicine is an international, peer-reviewed, open access, online journal publishing laboratory, experimental and clinical aspects of all aspect pertaining to blood based medicine including but not limited to: Transfusion Medicine; Blood collection, Donor issues, Transmittable diseases, and Blood banking logistics; Immunohematology; Artificial and alternative blood based therapeutics; Hematology; Biotechnology/nanotechnology of blood related medicine; Legal aspects of blood medicine; Historical perspectives. The manuscript management system is completely online and includes a very quick and fair peer-review system. Visit http://www.dovepress.com/testimonials.php to read real quotes from published authors. 